Supporting Information

\title{
Self-Assembled Nanostructures Based on Activatable Red Fluorescent Dye for Site-Specific Protein Probing and Conformational Transition Detection
}

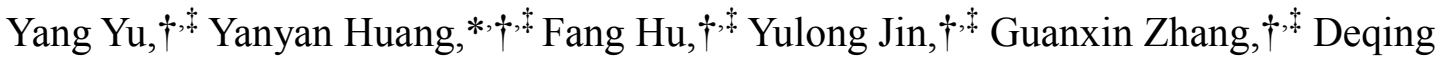 \\ Zhang, ${ }^{*, \dagger}$ Rui Zhao*,†,* \\ $\dagger$ Beijing National Laboratory for Molecular Sciences, CAS Key Laboratory of \\ Analytical Chemistry for Living Biosystems and Organic Solids, Institute of Chemistry, \\ Chinese Academy of Sciences, Beijing, 100190, China \\ $\$$ University of Chinese Academy of Sciences, Beijing, 100049, China \\ E-mail: zhaorui@iccas.ac.cn, yyhuang@iccas.ac.cn, dqzhang@iccas.ac.cn
}

\section{Table of content}

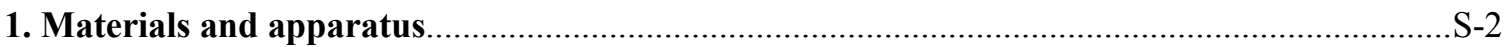

2. ${ }^{1} \mathrm{H}-\mathrm{NMR},{ }^{13} \mathrm{C}-\mathrm{NMR}$, FT-IR and MS characterization and HPLC analysis .............................. -3

3. Quantum yields of TPE-red-COOH in different solvents ...................................................

4. Fluorescence and dynamic light scattering (DLS) analysis of TPE-red-COOH........................

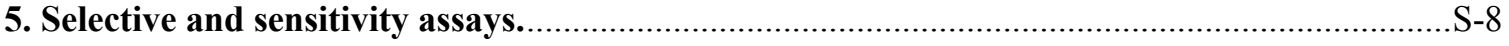

6. Investigation of the effects of bilirubin and ibuprofen to the fluorescence emission spectra of

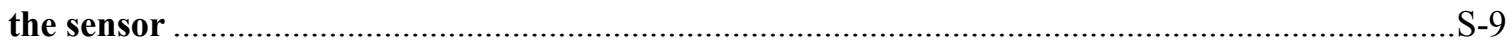

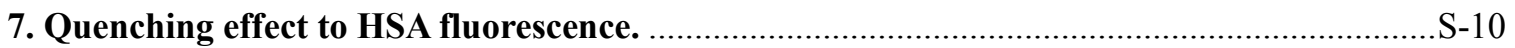

8. Effect of $\mathrm{pH}$ on the fluorescence response of the nanosenor to HSA ......................................

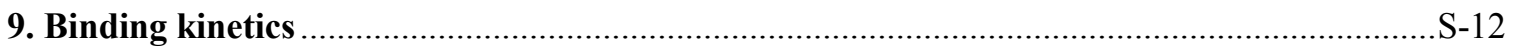

10. Fluorescence from Trp214 in HSA during unfolding and refolding processes. ................... -13

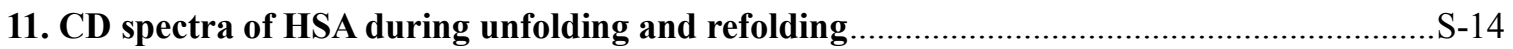




\section{Materials and apparatus}

Materials. HSA, bilirubin, trypsin, papain, vasopressin, glutathione (GSH), adenosine triphosphate (ATP) and guanidine hydrochloride $(\mathrm{GdnHCl})$ were purchased from Sigma-Aldrich (USA). Ibuprofen was purchased from National Institute for the Control of Pharmaceutical and Biological Products (Beijing, China). Glucosamine hydrochloride was from Chemical Reagent Factory (Shanghai, China). Chymotrypsin, pepsin and L-thyroxine were from Shanghai Institute of Biochemistry and Cell Biology (Shanghai, China). Methanol, acetonitrile and PBS ( $\mathrm{pH}$ 7.4) were purchased from ThermoFisher (USA). Tetrahydrofuran (THF), ethanol and dimethyl sulfoxide (DMSO) were of analytical grade and from Beijing Chemical Factory (China). Ultrapure water from a MilliQ water-purification system (Millipore, Bedford, MA, USA) was used throughout. Potassium carbonate were purchased from Alfa Aesar (USA) and used as received. For synthesis, all solvents were purified and dried following standard procedures.

Apparatus. Fluorescence measurements were carried out on a Hitachi F-4600 fluorometer (Tokyo, Japan). Absorption spectra were recorded on a Shimadzu UV-1800 spectrometer (Japan). ${ }^{1} \mathrm{H}-\mathrm{NMR}$ and ${ }^{13} \mathrm{C}-\mathrm{NMR}$ spectra were collected on a Bruker Avance $400 \mathrm{MHz}$ spectrometer using tetramethylsilane (TMS) as internal reference. HPLC analyses were performed on a Shimadzu Prominence UFLC system combined with a photo-diode array detector (Kyoto, Japan). Dynamic light scattering measurements were performed at $25{ }^{\circ} \mathrm{C}$ on a Zetasizer Nano ZS with a backscattering detection at $173^{\circ}$ and a $\mathrm{He}-\mathrm{Ne}$ laser (Malvern Instruments Ltd, UK). The morphology of the nano-aggregates was characterized by an HT7700 transmission electron microscope (Hitachi, Marunouchi, Japan). Time-resolved fluorescence experiments were carried out on a FLS980 combined with fluorescence lifetime and steady-state spectrometer (Edinburgh Co. Ltd., UK). Fourier transform infrared (FT-IR) spectroscopy characterization was performed with a Tensor-27 FT-IR spectrometer (Bruker Optics, Ettlingen, Germany). A total of 32 successive scans from each sample were collected at $4 \mathrm{~cm}^{-1}$ intervals and averaged 
spectra of each sample were used for analysis.

\section{2. ${ }^{1} \mathrm{H}-\mathrm{NMR},{ }^{13} \mathrm{C}-\mathrm{NMR}$, FT-IR and MS characterization and HPLC analysis}

a)

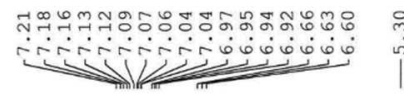

$$
\begin{aligned}
& \text { Jु } \\
& \text { Vं }
\end{aligned}
$$
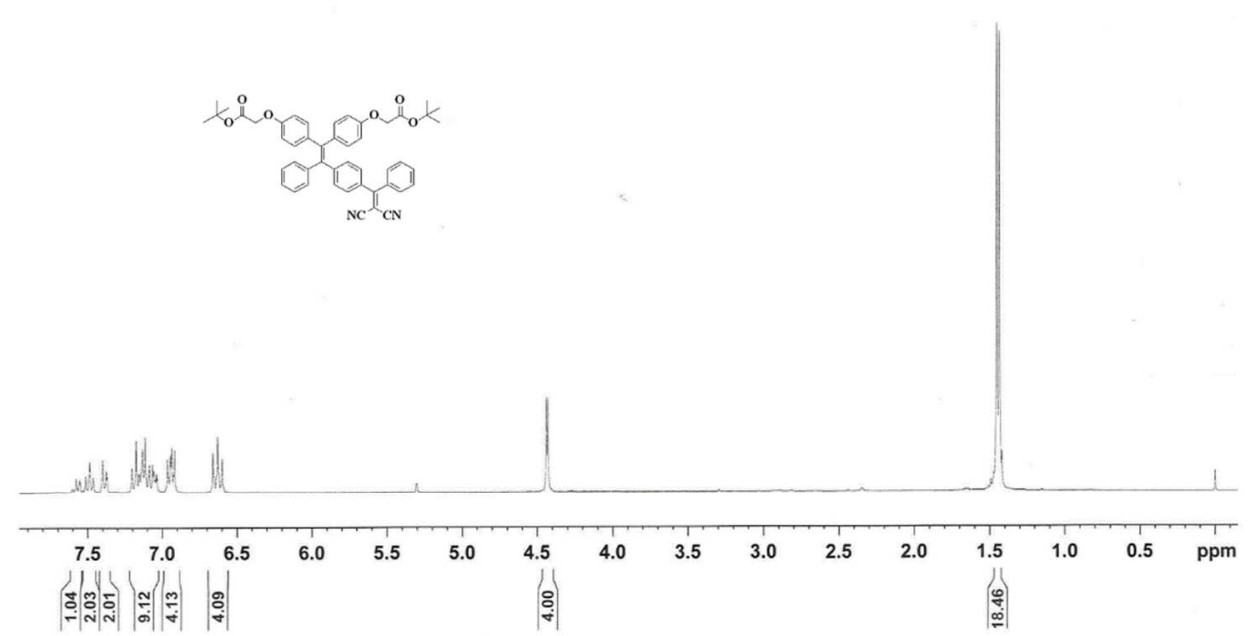

b)
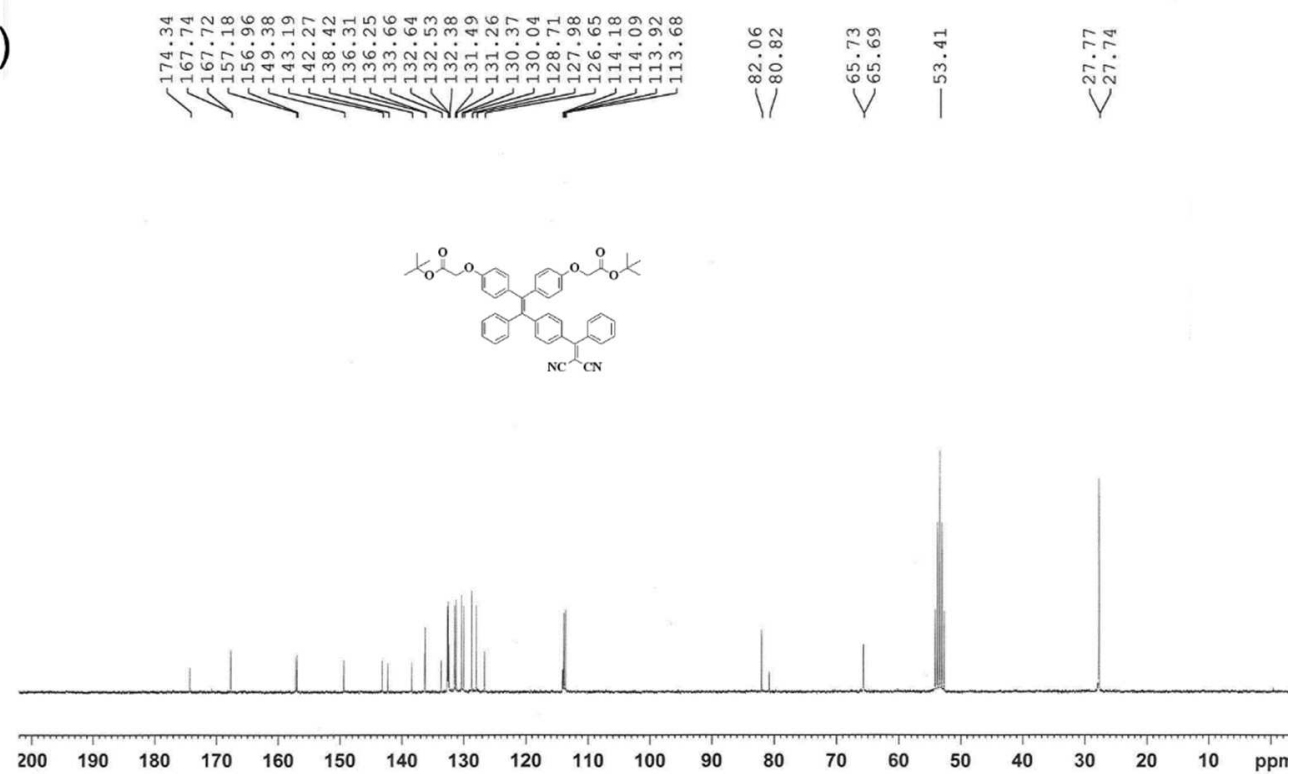

Figure S-1. (a) ${ }^{1} \mathrm{H}-\mathrm{NMR}$ spectrum of Compound 2 in $\mathrm{CD}_{2} \mathrm{Cl}_{2}$; (b) ${ }^{13} \mathrm{C}-\mathrm{NMR}$ spectrum of Compound 2 in $\mathrm{CD}_{2} \mathrm{Cl}_{2}$. 
a)

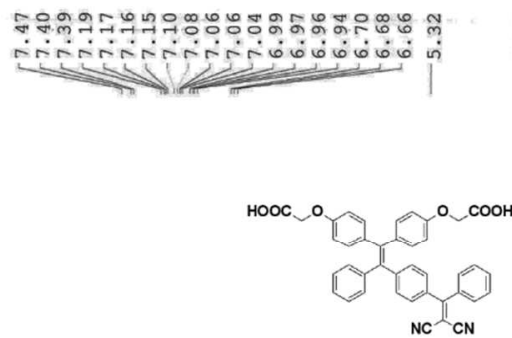

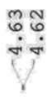

$\mathrm{C}^{\circ} \mathrm{CN}$

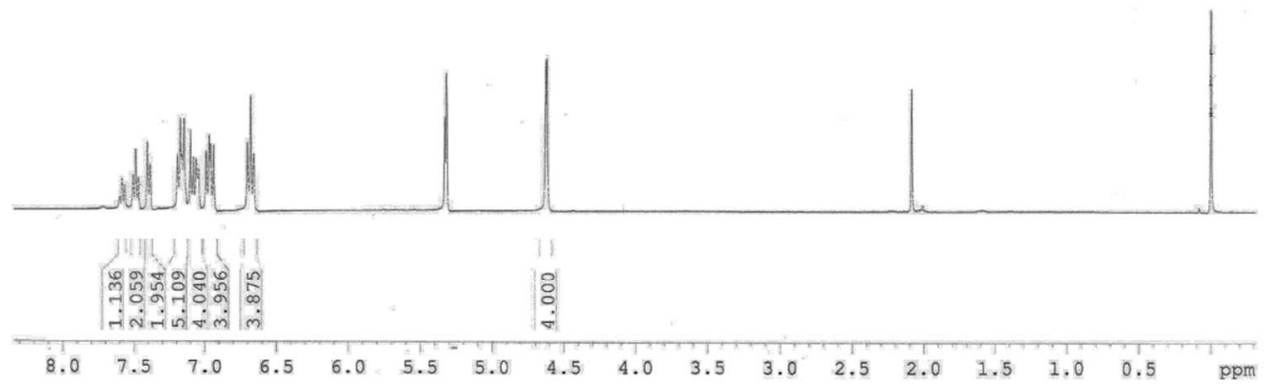

b)

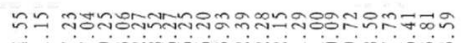

过

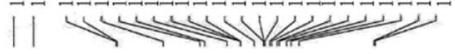
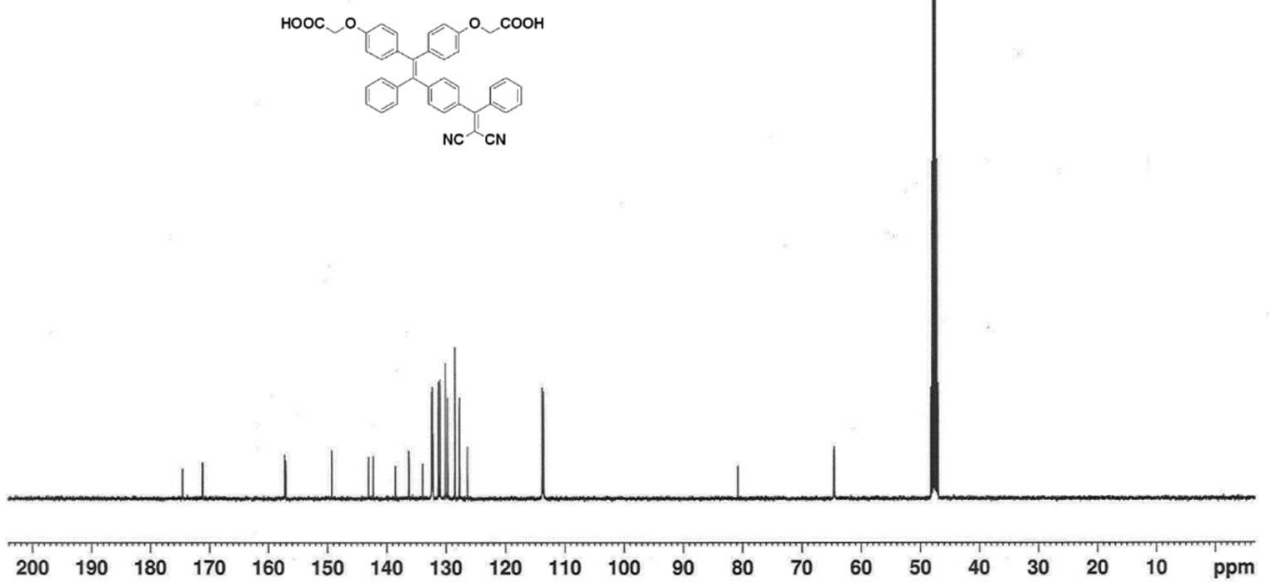

Figure S-2. (a) ${ }^{1} \mathrm{H}-\mathrm{NMR}$ spectrum of TPE-red-COOH in $\mathrm{CD}_{2} \mathrm{Cl}_{2}$; (b) ${ }^{13} \mathrm{C}-\mathrm{NMR}$ spectrum of TPE-red-COOH in $\mathrm{CD}_{3} \mathrm{OD}$. 
The FT-IR spectrum of TPE-red-COOH is shown in Figure S-3. The peaks at 3055, 3037 and $833 \mathrm{~cm}^{-1}$ can be attributed to the stretching vibration and bending vibration of $\mathrm{C}-\mathrm{H}$ bonds in the phenyl rings, respectively. The peak at $2924 \mathrm{~cm}^{-1}$ was attributed to the stretching vibration of $\mathrm{C}-\mathrm{H}$ bonds in methylene groups. The characteristic absorption band of the stretching vibration of $\mathrm{C} \equiv \mathrm{N}$ appeared at $2225 \mathrm{~cm}^{-1}$. The appearance of the band at $1733 \mathrm{~cm}^{-1}$ was caused by the stretching vibration of $\mathrm{C}=\mathrm{O}$. The peaks at 1500 and $1600 \mathrm{~cm}^{-1}$ were attributed to the stretching vibration of $\mathrm{C}=\mathrm{C}$ in the phenyl rings. The bands at 1220,1177 and $1076 \mathrm{~cm}^{-1}$ can be attributed to the stretching vibration of $\mathrm{C}-\mathrm{O}$. These results confirm the structural correctness of the synthesized product.

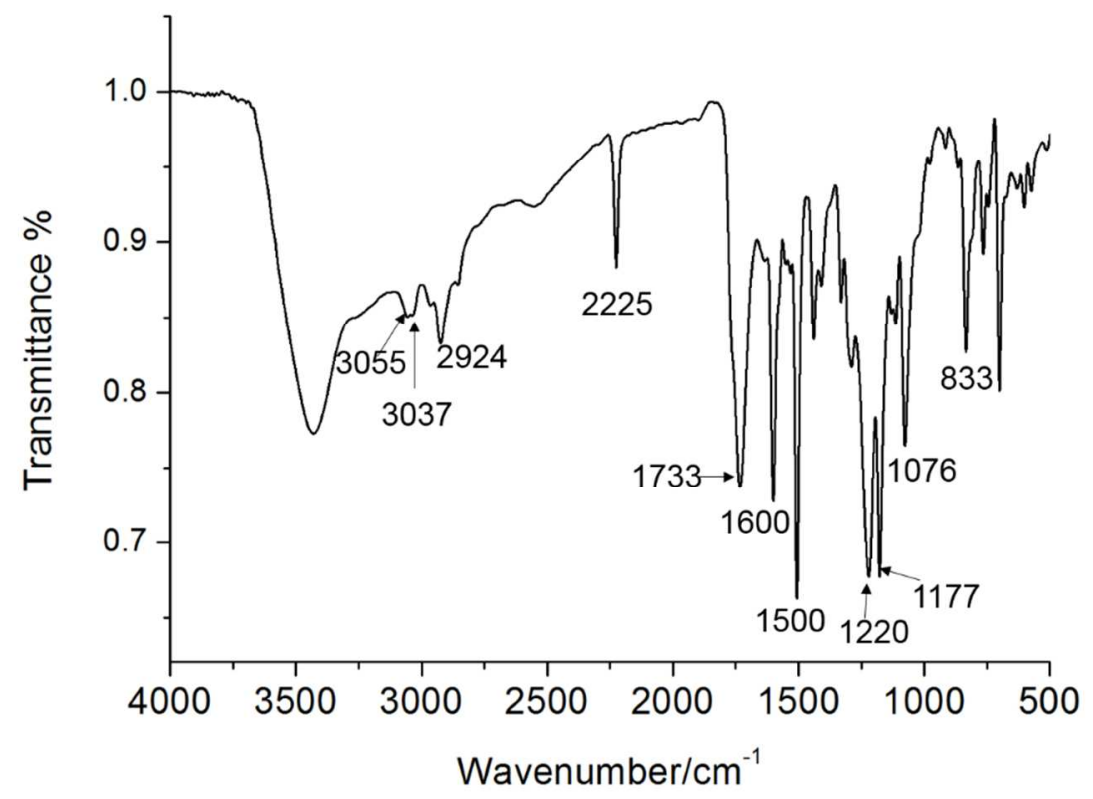

Figure S-3. FT-IR spectrum of TPE-red-COOH. 


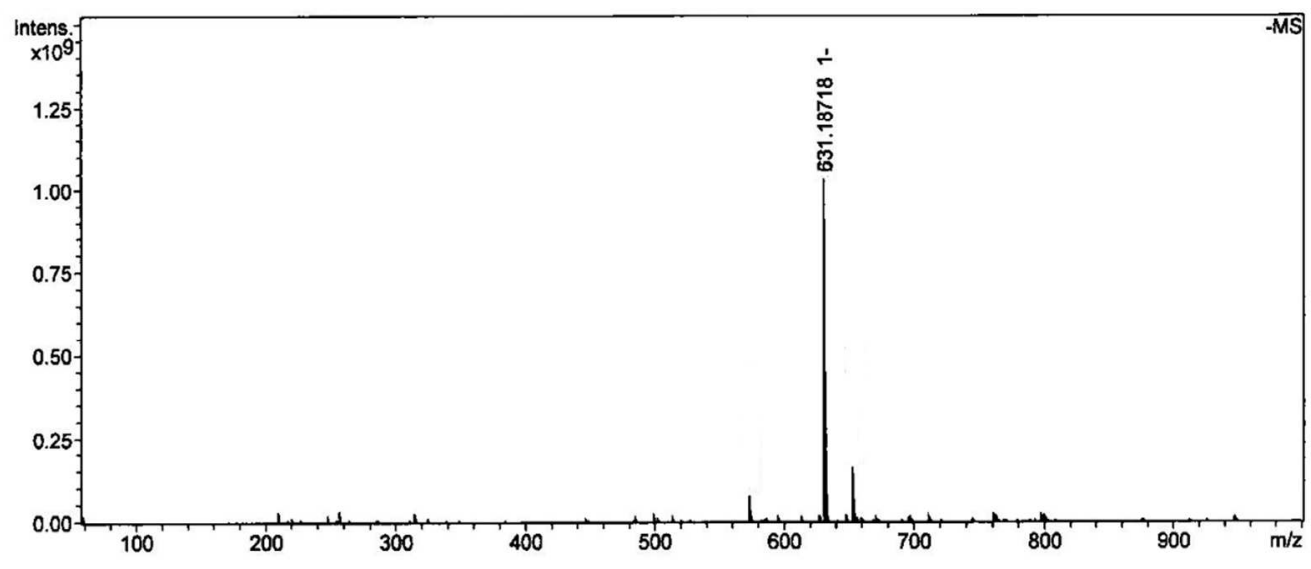

Figure S-4. High resolution mass spectrum of TPE-red-COOH operated in negative ion mode: HRMS (ESI, negative) m/z calcd: 631.18746 [(M-H)]; found: 631.18718.

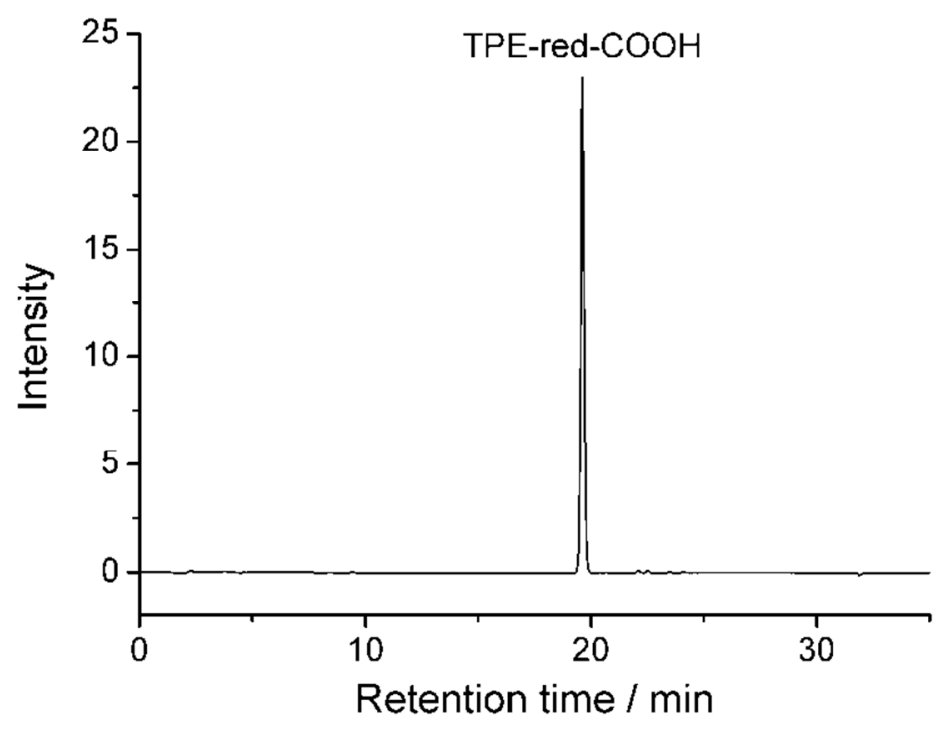

Figure S-5. HPLC analysis of TPE-red-COOH on a Dikma Diamonsil $\mathrm{C}_{18}$ column $(250 \times$ $4.6 \mathrm{~mm}$ ) monitored at the wavelength of $405 \mathrm{~nm}$. Solvent: $\mathrm{CH}_{3} \mathrm{CN}$. Gradient: 0-20 min30min, $60 \% \mathrm{~B}-100 \%-100 \% \mathrm{~B}\left(\mathrm{~A}: \mathrm{H}_{2} \mathrm{O}\right.$ containing $0.1 \%$ TFA, B: acetonitrile containing $0.1 \%$ TFA). 


\section{Quantum yields of TPE-red-COOH in different solvents}

The quantum yields of TPE-red-COOH in different solvents were measured according to the reported method. ${ }^{1}$ In PBS containing 20\% MeOH, 20\% EtOH and 20\% THF, the quantum yields of TPE-red-COOH were determined as $0.6 \%, 0.6 \%$ and $0.7 \%$ respectively using rhodamine $6 \mathrm{G}(\Phi=0.94)^{1}$ as the reference. In comparison, the quantum yields of TPE-red-COOH in PBS containing 1\% DMSO was only $0.09 \%$. These data demonstrated that the addition of $1 \%$ DMSO was the optimal solvent to prepare the fluorescence-silent nanosensor.

\section{Fluorescence and dynamic light scattering (DLS) analysis of TPE-red-COOH.}
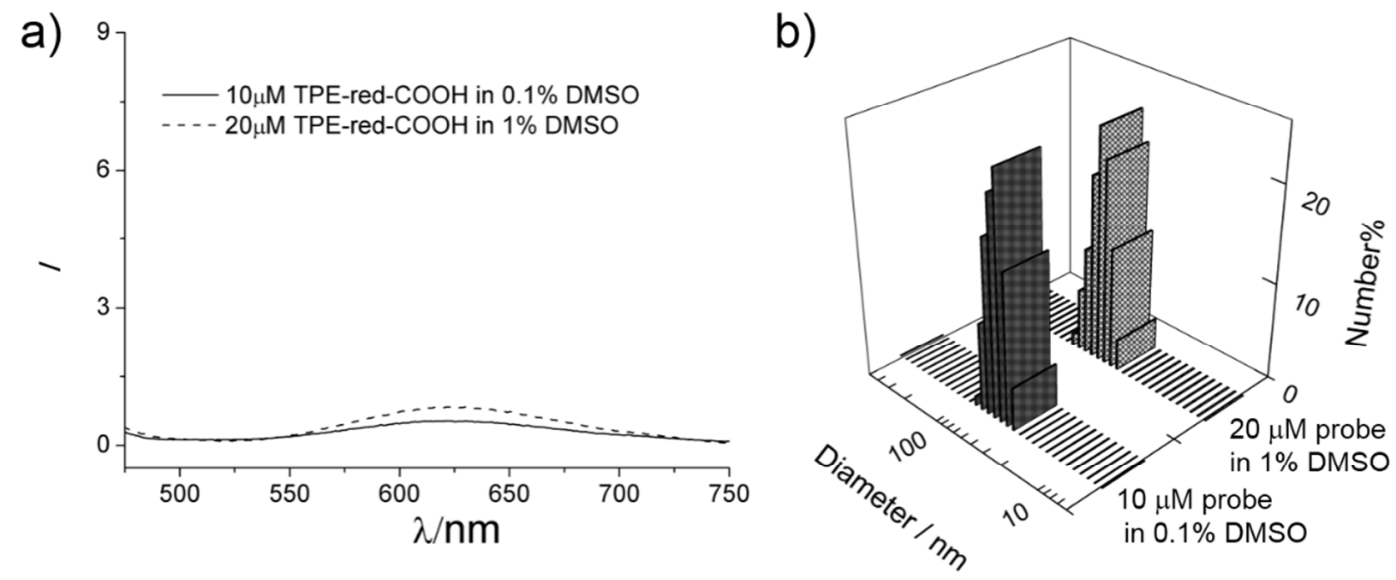

Figure S-6. (a) Fluorescence emission spectra and (b) DLS analysis of TPE-red-COOH with varied concentration of the probe and content of DMSO, $\lambda_{\mathrm{ex}}=405 \mathrm{~nm}$. 


\section{Selective and sensitivity assays.}

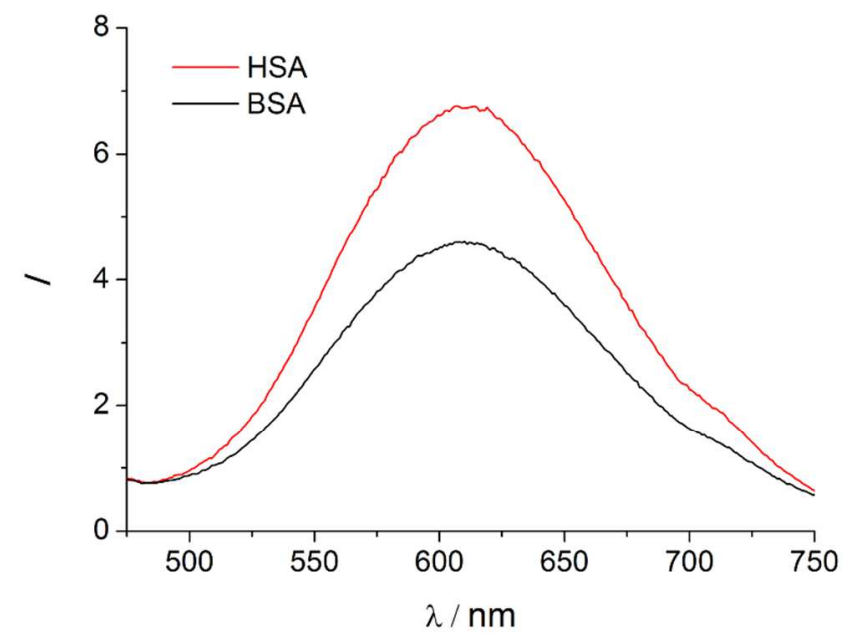

Figure S-7. Fluorescence emission spectra of TPE-red-COOH $(10 \mu \mathrm{M})$ with the addition of HSA and BSA (each $10 \mu \mathrm{M})$, respectively. $\lambda_{\mathrm{ex}}=405 \mathrm{~nm}$

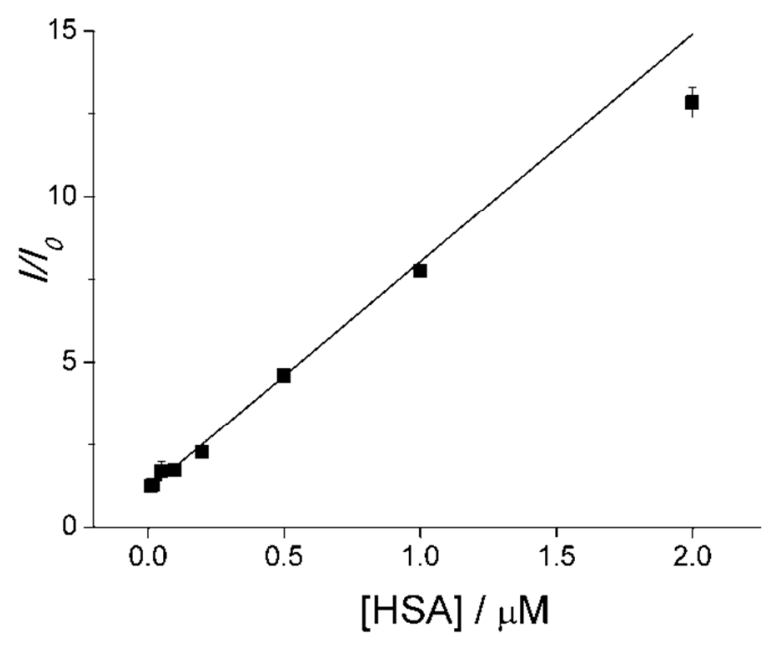

Figure S-8. The linear relationship between the fluorescence intensity and the concentration of HSA. $I$ and $I_{0}$ are the fluorescence intensities at $610 \mathrm{~nm}$ with and without the addition of HSA. $\lambda_{\mathrm{ex}}=405 \mathrm{~nm}$. 


\section{Investigation of the effects of bilirubin and ibuprofen to the fluorescence emission spectra of the sensor}

To examine the effect of bilirubin and ibuprofen on the fluorescence performance of the nanosensor, bilirubin and ibuprofen were mixed with the nanosensor, and the resultant fluorescence emission spectra were recorded. As shown in Figure S-9a, ibuprofen cannot induce any fluorescence change of the nanosensor even its concentration was increased to $100 \mu \mathrm{M}$ (9-fold higher than that of TPE-red-COOH). Similarly, the incubation with bilirubin also cannot lead to any fluorescence enhancement of the sensor (Figure S-9b). At higher concentrations of $50 \mu \mathrm{M}$ and $100 \mu \mathrm{M}$, the weak fluorescence signal in the range of 500-550 $\mathrm{nm}$ is most probably from bilirubin molecule itself, because similar fluorescence emission spectra were detected for bilirubin solutions with the corresponding concentrations (Figure S-9c). Based on these results, it can be concluded that bilirubin and ibuprofen cannot affect the fluorescence of the nanosensor. The fluorescence changes in the competitive assays are attributed to their different binding capabilities to HSA.
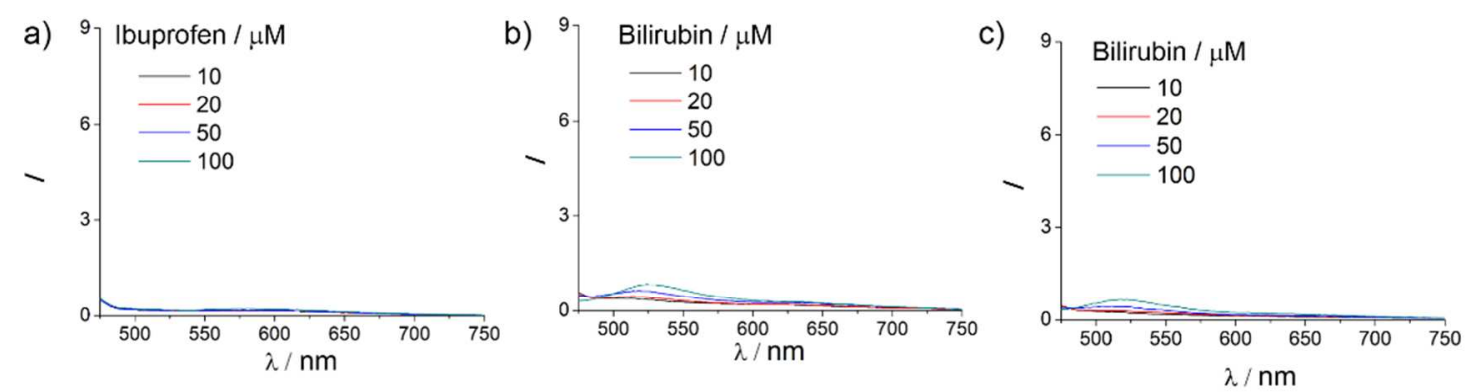

Figure S-9. Fluorescence emission spectra of TPE-red-COOH $(10 \mu \mathrm{M})$ responding to the addition of different concentrations of ibuprofen (a) and bilirubin (b), $\lambda_{\mathrm{ex}}=405 \mathrm{~nm}$. (c) Fluorescence emission spectra of different concentrations of bilirubin, $\lambda_{\mathrm{ex}}=405 \mathrm{~nm}$. 


\section{Quenching effect to HSA fluorescence.}

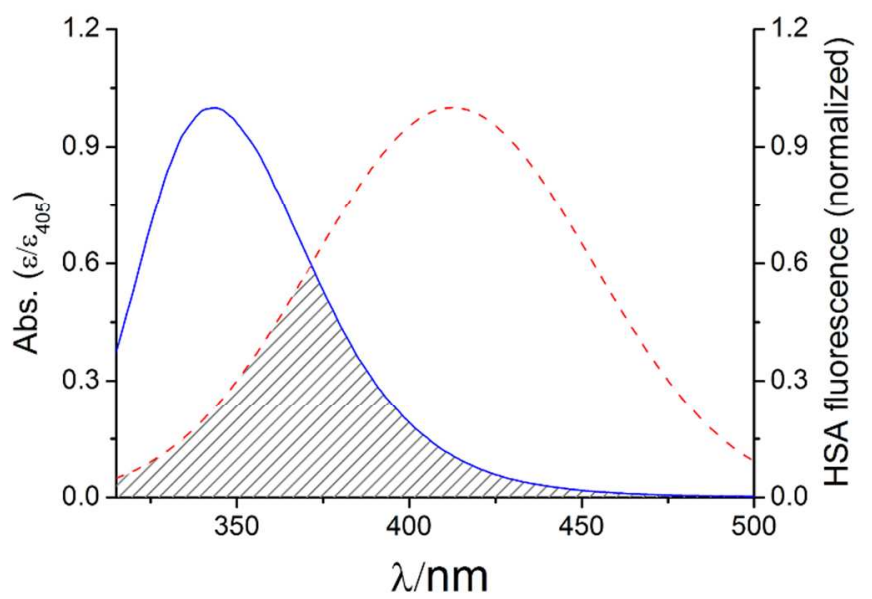

Figure S-10. Overlap of the fluorescence emission spectrum of HSA (blue, solid line) with the absorption spectrum of TPE-red-COOH (red, dashed line). $\lambda_{\mathrm{ex}}=295 \mathrm{~nm}$.

The quenching of HSA fluorescence by TPE-red-COOH can be described by Stern-Volmer equation

$$
F_{0} / F=1+k_{q} \tau_{0}[\mathrm{Q}]=1+K_{S V}[\mathrm{Q}]
$$

Where $F_{0}$ and $F$ are the fluorescence intensities in the absence and presence of the quencher respectively, $k_{q}$ is the quenching rate constant of the bimolecule, $\tau_{0}$ is the lifetime of the biomolecule in the absence of quencher, $K_{S V}$ is the Stern-Volmer quenching constant and $[\mathrm{Q}]$ is the quencher concentration.

By linear fitting $F_{0} / F$ versus [Q] at $25^{\circ} \mathrm{C}$ (Figure S-11), the quenching rate constant $k_{q}$ was calculated to be $1.08 \times 10^{13} \mathrm{M}^{-1} \mathrm{~s}^{-1}$. This value is higher than the limiting diffusion rate constant of various kinds of quenchers with biomolecules $\left(K_{d i f}=2.0 \times 10^{10} 1 \mathrm{~mol}^{-1}\right.$ $\mathrm{s}^{-1}$ ), manifesting that a static quenching mechanism is operative for the complex formed by TPE-red-COOH and HSA., ${ }^{2,3}$ 


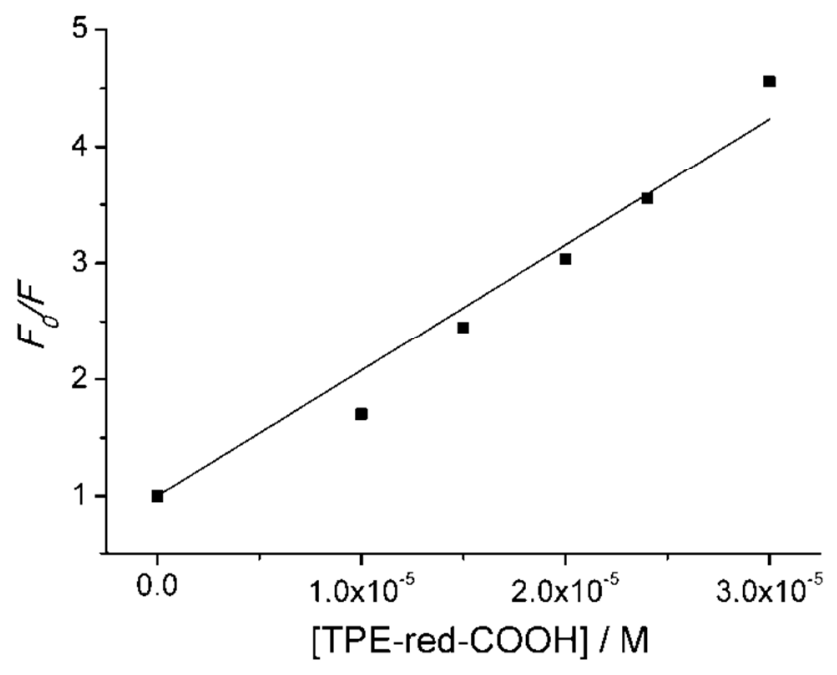

Figure S-11. Plot of $F_{0} / F$ versus the concentration of TPE-red-COOH. The concentration was fitted at $10 \mu$ M. $\lambda_{\text {ex }}=295 \mathrm{~nm}$.

\section{Effect of $\mathrm{pH}$ on the fluorescence response of the nanosenor to HSA}

The fluorescence response of the nanosensor to HSA was also examined under different $\mathrm{pH}$ conditions. As shown in Figure S-12, the relative fluorescence intensity of the nanosensor at $610 \mathrm{~nm}$ elevates as the $\mathrm{pH}$ value increases, suggesting enhanced interaction under basic condition. Since $\mathrm{pH}$ is the key factor determining the protonating and deprotonating status of the carboxylic groups in TPE-red-COOH and amino groups in $\mathrm{HSA}$, the change in $\mathrm{pH}$ poses direct effect on the electrostatic interactions between TPE-red-COOH and amino groups in HSA. Under acid conditions, TPE-red-COOH molecules in the nanosensor are protonated and difficult to generate electrostatic forces with HSA. With higher $\mathrm{pH}$, TPE-red-COOH nanosensor undergoes deprotonating and carries more negative charges. As a result the nanosensor forms stronger electrostatic interactions with the basic residues such as Lys195 and Lys199 on the surface of HSA. Additionally, the amino side chains of these basic residues in HSA are all positively charged at $\mathrm{pH}$ 7.4. Therefore, it is easy to form cation- $\pi$ interaction between these surface-located basic residues in HSA and TPE-red-COOH backbone. The combination 
of electrostatic and cation- $\pi$ interactions may play as the critical roles for the disassembly of the nanosensor.

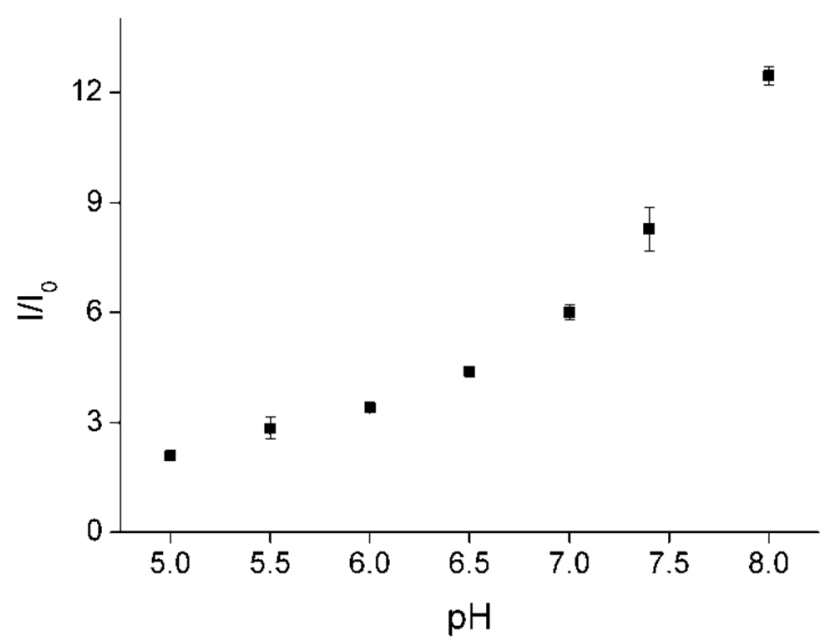

Figure S-12. Effect of $\mathrm{pH}$ on the fluorescence response of the nanosensor to HSA. I and $I_{0}$ are the fluorescence intensities at $610 \mathrm{~nm}$ of the nanosensor with and without the addition of HSA under various $\mathrm{pH}$.

\section{Binding kinetics}

The binding kinetics of TPE-red-COOH towards HSA was examined by recording the fluorescence emission at $610 \mathrm{~nm}$ after different incubation periods. As shown in Figure S-13, the fluorescence intensity reached the maximum only with 5-min incubation and kept constant was time elongated. These results suggest the fast binding between TPE-red-COOH and HSA. 


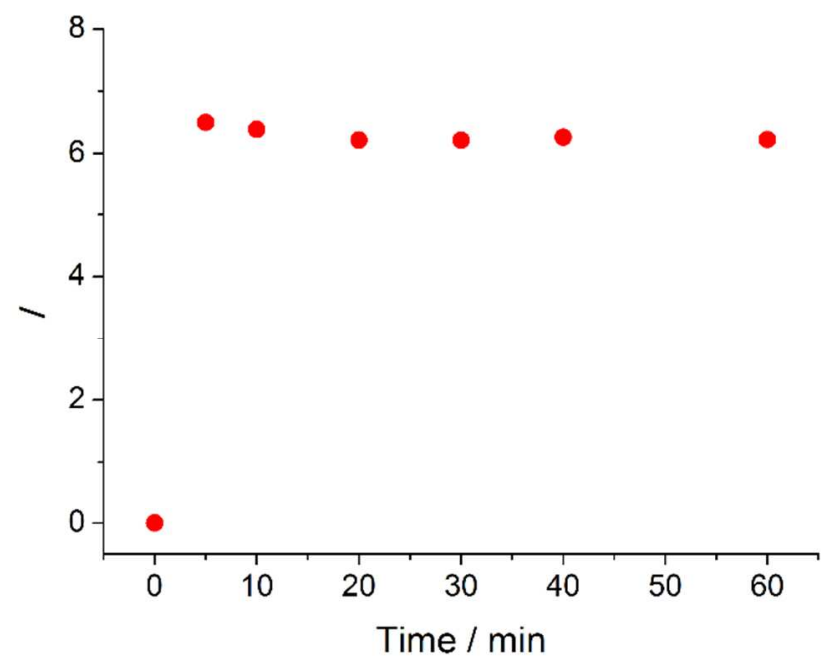

Figure S-13. Fluorescence response of the nanosensor after incubating with HSA for various time intervals. $\lambda_{\mathrm{ex}}=405 \mathrm{~nm}, \lambda_{\mathrm{em}}=610 \mathrm{~nm}$.

\section{Fluorescence from Trp214 in HSA during unfolding and refolding processes.}

The native HSA shows typical emission at $340 \mathrm{~nm}$, representing that Trp214 is buried in hydrophobic interior of the protein (Figure S-14). After the treatment of $3 \mathrm{M} \mathrm{GdnHCl,} \mathrm{the}$ peak position show negligible movement. This phenomenon indicates that Trp214 residue still remained buried in the Sudlow site I pocket under this condition, because the fluorescence peak of tryptophan is very sensitive to environmental polarity. ${ }^{4,5}$ With the addition of $6 \mathrm{M} \mathrm{GdnHCl}$, a distinct red-shift of emission peak was detected (Figure S-14). The peak at $350 \mathrm{~nm}$ is attributed to the tryptophan emission in polar microenvironment. This result suggests that $6 \mathrm{M} \mathrm{GdnHCl} \mathrm{leads} \mathrm{to} \mathrm{the} \mathrm{unfolding} \mathrm{of} \mathrm{HSA} \mathrm{and} \mathrm{exposure} \mathrm{of}$ Trp214 to the aqueous solvent. ${ }^{2}$ 


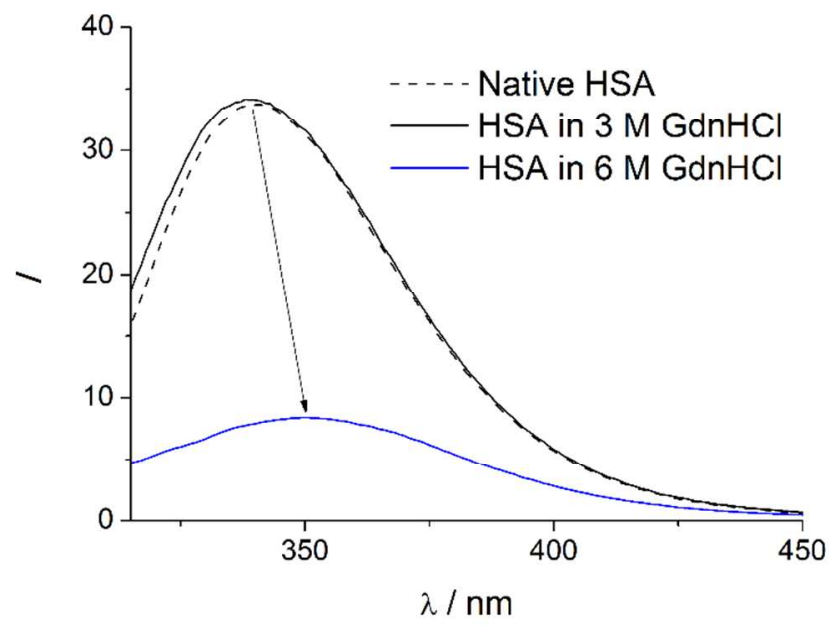

Figure S-14. Fluorescence emission spectra of HSA $(10 \mu \mathrm{M})$ with the addition of different concentrations of $\mathrm{GdnHCl}$. $\lambda_{\mathrm{ex}}=295 \mathrm{~nm}$.

\section{CD spectra of HSA during unfolding and refolding}

Circular dichroism (CD) spectroscopy was employed for monitoring the conformational changes of HSA during unfolding and refolding. Native HSA shows a typical $\alpha$-helix profile in CD spectrum with two characteristic signals at $208 \mathrm{~nm}$ and $220 \mathrm{~nm}$ (Figure S-15).

During the denaturing process, with increasing concentrations of $\mathrm{GdnHCl}$ ranging from 0.1 to $2 \mathrm{M}$, the CD signals almost kept unchanged (Figure S-15a). This is attributed to the fact that $\mathrm{CD}$ is a powerful tool for characterizing secondary structures of protein, however is not able to provide information on three dimensional or the tertiary structures. ${ }^{6}$ The CD spectra suggest that the secondary structure of HSA was not affected by low concentrations of $\mathrm{GdnHCl}$. In comparison, due to its site-specific binding behavior and structure-sensitive feature, the nanosensor can detect the change in the three dimensional structure of HSA induced by low concentrations of $\mathrm{GdnHCl}$. At higher concentrations of $\mathrm{GdnHCl}$, significant decrease in $\mathrm{CD}$ signal was detected (Figure S-15a, b), suggesting the loss of the secondary structure and unfolding of HSA. Such phenomenon is in accordance with the fluorescence decrease of the nanosensor. 

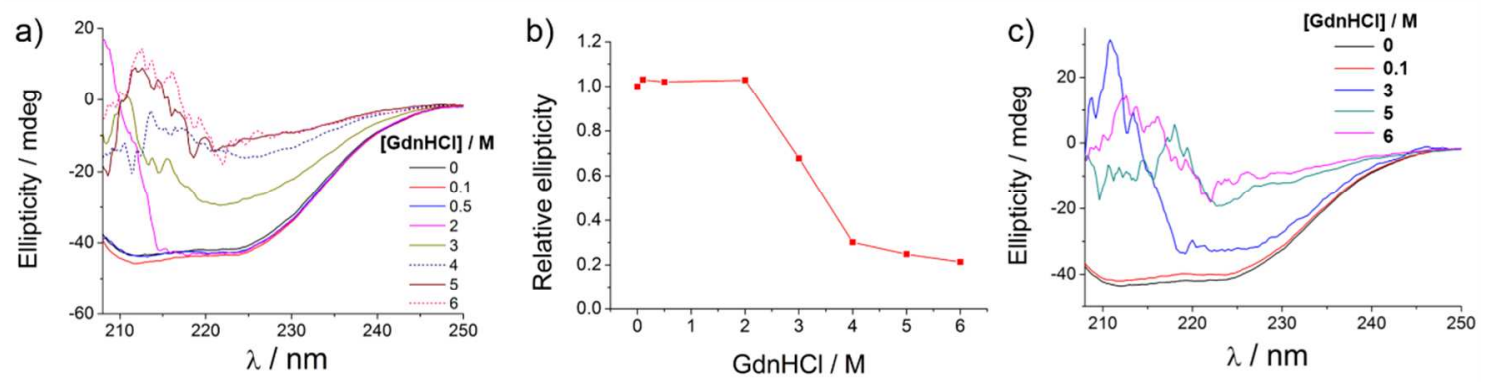

Figure S-15. (a) CD spectra of HSA in PBS containing different concentrations of GdnHCl. (b) Plot of the relative ellipticity of HSA at $220 \mathrm{~nm}$ versus $\mathrm{GdnHCl}$ concentration during denaturation process. (c) CD spectra of HSA during refolding process.

CD spectra were also recorded during the refolding process (Figure S-15c). With gradually diluted concentration of $\mathrm{GdnHCl}, \mathrm{CD}$ signal was recovered. At the $\mathrm{GdnHCl}$ concentration of $0.1 \mathrm{M}$, the $\mathrm{CD}$ spectral data show $95 \%$ recovery in the secondary structure. Similarly, the fluorescence signal from the nanosensor was $97 \%$ restored after the removal of $\mathrm{GdnHCl}$.

\section{References}

(1) Brouwer A. M. Pure Appl. Chem. 2011, 83, 2213-2228.

(2) Abou-Zied, O. K.; Al-Shihi, O. I. K. J. Am. Chem. Soc. 2008, 130, 10793-10801.

(3) Ware, W. R. J. Phys. Chem. 1962, 66, 455-458.

(4) Kamal, J. K.; Zhao, L.; Zewail, A. H. P Natl Acad Sci USA. 2004, 101, 13411-13416.

(5) Kamal, J. K. A.; Behere, D. V. Biochemistry-US. 2002, 41, 9034-9042.

(6) Ahmad, B.; Ahmed, M. Z.; Haq, S. K.; Khan, R. H. Biochim. Biophys. Acta Protein Proteomics. 2005, 1750, 93-102. 